\title{
Fabrication and characterization of a Gallium co-doped Erbium optical fiber
}

\begin{abstract}
In this paper, fabrication and characterization of a Gallium co-doped Erbium fiber is presented, highlighting Gallium as a new potential co-dopant to be used in rare-earth doped fibers. This fiber was fabricated using standard MCVD and solution doping method. Fiber characterization setups for fluorescence lifetime, absorption and ASE spectrum are discussed in detail. We go on to show that fluorescence lifetime of $6.02 \mathrm{~ms}$, NA of 0.12 , cutoff wavelength of $1.4 \mathrm{Em}$ and a peak absorption of $45 \mathrm{~dB} / \mathrm{m}$ at $1550 \mathrm{~nm}$ is achievable using Gallium as the co-dopant for an Erbium doped fiber.
\end{abstract}

Keyword: Absorption spectrum; ASE spectrum; Erbium; Fluorescence lifetime; Gallium; MCVD; Optical fiber; Rare-earth doped fiber; Solution doping 